\title{
STAR-FORMING ACTIVITY IN ARP-MADORE GALAXIES
}

\author{
A.M. HOPKINS AND L.E. CRAM \\ University of Sydney NSW 2006 \\ Australia
}

\section{Introduction \& method}

The rate of star formation is enhanced in many galaxies that show disturbed morphology and/or evidence of interaction. However, the physical explanation of this connection has proved elusive, since it appears that peculiar morphology of a specific type is neither necessary nor sufficient to promote star formation. To explore the relation between star formation rates and galaxy morphology we have selected a sample of galaxies from the Arp-Madore Catalogue of Southern Peculiar Galaxies and Associations (AMC) and the Principle Galaxy Catalogue (PGC), and estimated their star formation rate using the far-infra-red (FIR) power listed in the $Q M W$ IRAS Galaxy Catalogue (QMCIGC). There are 933 objects common to all three catalogues, potentially providing the necessary data for the peculiar galaxies.

We also identified a subset of 'normal' galaxies from the PGC and QMCIGC containing 523 objects to form a sample matched to the AM objects by absolute blue luminosity, absolute diameter, $\mathrm{H}$ I line width and redshift. With the exception of the redshift distribution, the two sample distributions were identical as characterized by the Kolmogoroff-Smirnov test. The redshift distribution of the 'normal' sample lies at smaller values than the $\mathrm{AM}$ galaxies, reflecting the relative rarity and possibly the intrinsic interest of AM-type objects.

From these samples we selected all of the objects having published blue (in PGC) and FIR (in QMCIGC) luminosities. We then determined a number of astrophysically significant quantities for the samples, including the ratio of FIR to blue luminosity as a measure of the relative rate of star formation. After inspecting the distribution of this ratio, we determined that if the ratio is greater than $10^{0.3}$, the galaxy may be very actively forming 
stars. Table 1 illustrates the propensity for galaxies from either sample to be an actively star forming object.

TABLE 1. Distribution of $\log (\mathrm{FIR} / \mathrm{B}-\mathrm{band})$

\begin{tabular}{ccc}
\hline $\log ($ FIR/B-band) & AM sample (\%) & Control sample (\%) \\
\hline$>0.3$ & 0.125 & 0.019 \\
$>0.2$ & 0.132 & 0.030 \\
$>0.0$ & 0.154 & 0.041 \\
\hline
\end{tabular}

\section{Results \& Conclusions}

There are $51 / 408$ or $12.5 \%$ of the AM sample classified as active, and $7 / 370$ or $1.9 \%$ of the control sample so classified. We conclude that AM galaxies are more likely to exhibit star-forming activity than galaxies without peculiarities. Of the Arp-Madore morphological categories, the ones with the highest proportion of activity belong to categories such as AM9 (M51-type with a companion at the end of a spiral arm) which are "recent" or "early" interactions. These are not the galaxies having the greatest tidal distortion. Our result suggests that increased star-forming activity sets in early in the interaction, and has faded by the time that tidal effects are fully manifest.

On the other hand, the great majority of AM objects show little or no sign of excess star forming activity. Also, some of the control sample galaxies have high rates of star formation but are not associated with the type of peculiarity leading to selection in the AM catalogue.

We considered differences between the AM sample and the control sample for subsamples selected (1) to be known Seyfert galaxies and (2) to be known members of ACO clusters. There is no significant increase in star-forming activity in the AM Seyferts (18 objects) relative to the control Seyferts (63 objects). There is a larger fraction of the AM galaxies $(64 / 933=6.9 \%$ versus $9 / 523=1.7 \%)$ in clusters, and of the cluster members the activity is notably higher in the AM galaxies although only one has an index greater than 0.3 .

The relatively large proportion of M51-type galaxies found to be among the most active AM objects is consistent with the occurrence of star formation at an early phase of an interaction. The proportion of AM galaxies showing a high rate of star formation is smaller for objects with well developed evidence of tidal interaction. This is consistent with the idea that star-forming time scales are of the order of $10^{8}$ years, while tidal interaction time scales are of the order of $10^{9}$ years. 